\title{
The Secrets of a Functional Synapse - From a Computational and Experimental Viewpoint Michal Linial*
}

Address: Dept of Biological Chemistry, The Hebrew University of Jerusalem, 91904, Israel

Email: Michal Linial* - michall@cc.huji.ac.il

* Corresponding author

from NIPS workshop on New Problems and Methods in Computational Biology

Whistler, Canada. 18 December 2004

Published: 20 March 2006

BMC Bioinformatics 2006, 7(Suppl I):S6 doi:I0.I I86/I47I-2105-7-SI-S6

\begin{abstract}
Background: Neuronal communication is tightly regulated in time and in space. The neuronal transmission takes place in the nerve terminal, at a specialized structure called the synapse. Following neuronal activation, an electrical signal triggers neurotransmitter (NT) release at the active zone. The process starts by the signal reaching the synapse followed by a fusion of the synaptic vesicle and diffusion of the released NT in the synaptic cleft; the NT then binds to the appropriate receptor, and as a result, a potential change at the target cell membrane is induced. The entire process lasts for only a fraction of a millisecond. An essential property of the synapse is its capacity to undergo biochemical and morphological changes, a phenomenon that is referred to as synaptic plasticity.

Results: In this survey, we consider the mammalian brain synapse as our model. We take a cell biological and a molecular perspective to present fundamental properties of the synapse:(i) the accurate and efficient delivery of organelles and material to and from the synapse; (ii) the coordination of gene expression that underlies a particular NT phenotype; (iii) the induction of local protein expression in a subset of stimulated synapses. We describe the computational facet and the formulation of the problem for each of these topics.
\end{abstract}

Conclusion: Predicting the behavior of a synapse under changing conditions must incorporate genomics and proteomics information with new approaches in computational biology.

\section{Introduction}

The structure and function of the synapse has been the topic of extensive research for many decades [1-5]. It is only in recent years that the molecular complexity of this structure has been fully appreciated. The availability of complete genomes throughout the evolutionary tree raised new avenues to address the link between the molecules that are involved in the structural organization of the synapse and its functionality [6-8].

\section{Computational Neuroscience}

Most research in the field of computational neuroscience can be viewed in a 'systems biology' perspective, meaning that each of the neurons is considered to be an integrator device and a major goal is to understand the behavior of the neuronal network. For such a task, strong theoretical tools, combined with experimental measurements, proved to be essential. Still, due to the inherent complexity of the mammalian CNS, much of the research in this 
field focuses on the fairly simple neuronal circuits of invertebrates [9-11]. A desirable goal is to develop a simulator that will accurately predict neuronal network properties (such as synchronization, rhythm, robustness). Of course, a key component in building such a computational scheme is to incorporate the biochemical and biophysical properties of the neuron. Fortunately, accurate techniques were developed over the years that allow direct measurements of neurons in-vivo and in-vitro with high spatial and temporal resolution, including subcellular resolution imaging of $\mathrm{Ca}^{2+}$ dynamics, electrophysiological measurements of a single ion channel and more.

As a result of the sequencing of the human genome and the genomes of hundreds of other organisms, many genomics and proteomics databases and tools that are specific to the field of neuroscience are becoming available [12-15]. The outcome of recent large-scale genomics and proteomics measurements (i.e. DNA-, Protein- and Cell-Arrays) is a quantitative view on the quantities, subtypes, interactions and modifications of all components in the cell, including molecules that determine neuronal functionality (such as ion channels, transporters, receptors, and protein kinases). Additional technologies that complement the above data are time-lapse subcellular visualization techniques and systematic genetic intervention (i.e., RNAi screen, [16]). It is expected that when all these data sources are integrated at the cellular level, the construction of an accurate quantitative model of a functional neuronal network becomes feasible. A long term application for these powerful techniques is in deciphering the molecular basis of neurological and mental diseases [17].

Having briefly described some of the development in the field of genomics and proteomics, we would like to go back to the functional synapse. A functional synapse can be described in terms of its expressed molecular information (i.e., transcripts, proteins). This paper aims to cover few selected topics in cellular neuroscience. In order to appreciate the context, we provide the elementary background needed to understand the functionality of the synapse. We choose to address three different aspects related to a mature CNS synapse: (i) the principles underlying organized delivery of material in the axons; (ii) the coordinated expression of functionally related genes; and (iii) trafficking and translational control outside of the cell body. For each of the topics, we will touch upon the computational biology questions that arise and propose potential directions to address these questions. Due to the space constrains, we mostly cite review articles rather than primary sources, and we limit our discussion to the level of a single synapse.

\section{Neuron anatomy and physiology}

For a brief introduction to nerve cell anatomy, we recall the following facts and principles of a neuron's anatomy. The mammalian central nerve system (CNS) is made up of a hundred billions nerve cells. Typically, a neuron contains (i) a central cell body that directs all activities of the neuron; (ii) dendrites that receive messages from other neurons and relay them to the cell body; (iii) an axon, a long fiber that transmits messages from the cell body to the dendrites of neighboring neurons. The synapses of the CNS and those of the peripheral nerve system share many anatomical and functional features. Yet, they are markedly different in their plasticity. For simplicity, we will focus on a prototype mammalian CNS synapse as our model.

The communication between nerve cells is based on the detailed structures and organization of the transmitting axon and of the receiver dendrite. These structures and the space between them comprise the synapse. According to the directionality of the transmission, the axon terminal is denoted as the presynaptic site while the dendrite comprises the postsynaptic site $[1,18,19]$. When neurons communicate, an electrical impulse propagates down the axon and toward the synapse. There, following depolarization and influx of $\mathrm{Ca}^{2+}$, synaptic vesicles (SVs) are fused, resulting in a release of NT to the synaptic cleft. The NT diffuses across the synaptic cleft and binds to the appropriate NT receptors that are located within the postsynaptic membranes of the adjacent cell. This, in turn, stimulates (or inhibits) an electrical response. The presynaptic site is rapidly recovers, i.e., SVs are recycled and refilled. This sequence of events define a basic functional step of any synapse.

\section{A. The Neuronal Anatomy}

Many properties of neuronal function are attributed to the cell's anatomy. Neuronal anatomy is tightly linked to the inherent polarity of neurons. We will not discuss the mechanism underlying the establishment of cell polarity $[20,21]$. However, we will dwell on the difficulty of maintaining and supporting a cell with such an extreme polarity. In most neurons, the axon that extends from the cell body is very far from the site of synapse, where NT release takes place: while the size of an average synapse is only a few microns, the neuronal cell body may be as far as one meter or more from its nerve terminal. While electrical information can be transferred through the membrane very quickly, this is not the case for the chemicals, proteins, membranes and organelles that are to be shipped back and forth between the cell body and the nerve terminal. The cell body must constantly supply new materials to the synapse. This includes proteins for NT storage, metabolic enzymes for NT production or its breakdown, resident proteins of SVs, regulators of exo- and endocytosis, 
growth factors and neurotrophins, mitocondria and more.

Protein translation, peptide and hormone storage and formation of new membranes are carried out in the cell body (and to some extend also in the dendrites, see section $\mathrm{C}$ ). As no local protein production is possible in nerve terminals, all material must be coordinately transported. The anatomical difficulty has to be resolved as the synapse must respond to signals from its surrounding, and to messages coming from the cell body.

What are the cellular and molecular strategies that are adopted by the neuron for an efficient and accurate delivery between the two remote subcellular entities? Transport of membranes, organelles and proteins to the synapse is referred to as axonal transport. Extensive studies of axonal transport shed light on its molecular basis: the microtubules (MT) are the 'freeways' on which the molecular motors act as vehicles that deliver their cargo. Note that a retrograde transport from the nerve terminal to the cell body also exists. The fundamental properties of axonal transport are as follows: (i) energy drives the process; (ii) the tracks for movement are the axonal MT; (iii) movement is accomplished by a family of motor proteins called kinesins; (iv) most material is delivered by transport vesicles. Similar principles for MT dependent movements are found outside of the nerve system [22].

Kinesin molecules show a large variation in their biophysical properties. Nevertheless, a generic member of kinesin is composed of a motor domain that provides the movement, a connector domain that determines the properties and directionality of the movement elementary step, and a tail domain that is in charge of the specificity to the transported cargo (Fig. 1). Based on sequencing and on the annotation of the human and some rodent genomes, it becomes clear that the molecular complexity of the kinesin family is higher than anticipated [23-25]. The 41 predicted sequences in the human genome (48 in the mouse genome) are classified into 14 subfamilies with some additional orphan members [26]. Most kinesins are similar in their domain organization. Experimental evidences suggest the each kinesin provides its own uniqueness in term of the biophysical properties (i.e speed, efficiency, step size and association with the MT track) and the specificity toward the cargo [27]. For example, a particular kinesin transports mitochondria while a closely related member is associates with SV transport.

From the biological perspective, the issue of specificity and redundancy among the 48 kinesins in the mouse genome is still open. The hypothesis that the number of kinesin molecules and their composition on a transport vesicle deterministically define the capacity of the neuron

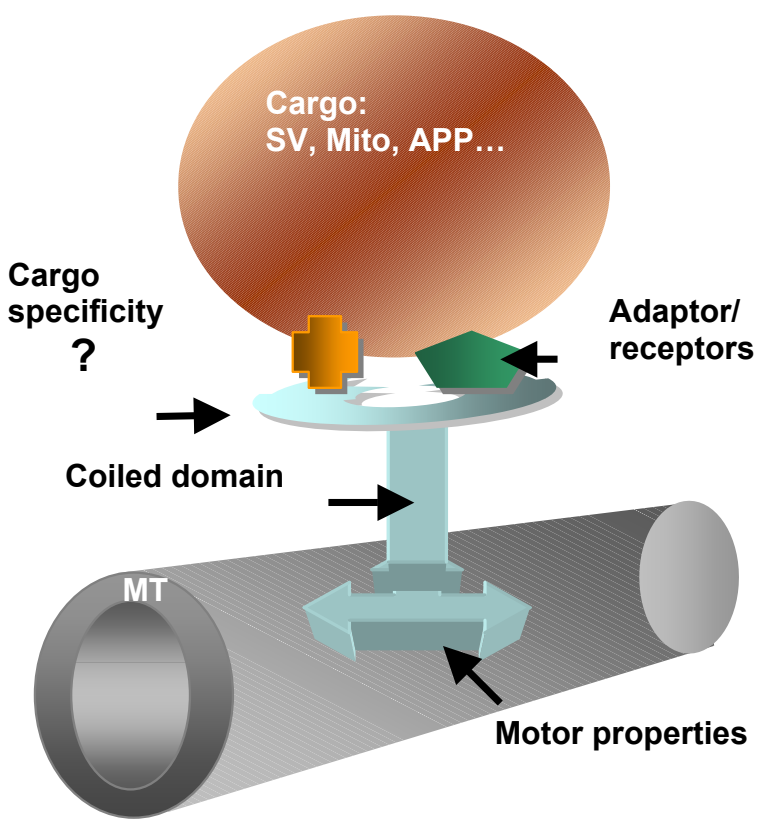

Figure I

A scheme for kinesin attached to mictotubules (MT) and to a vesicle. The molecule is composed of two heads (marked by an arrow) that allow attachment to MT, a central coiled region and a region which connects the molecule to the intracellular vesicle to be moved. The movement is based on ATP hydrolysis by the head motor domain. Adaptor and receptors associated with the cargo vesicles (SV, synaptic vesicle; Mito, mitochondria; APP, Amyloid precursor protein) provide another strategy to enrich motor-cargo combinations.

to replenish the synapse remains to be tested. The specificity of the different kinesins cannot be based on lipid recognition but rather on a very specific interaction with adaptor proteins and with membrane associated recognition complexes (Fig. 1). The confirmation that an intermediate molecule links a kinesin motor to its cargo vesicle allows a richer combinatorial 'mix and match' strategy for transport. Indeed, it was shown that KIF5, with the help of linker molecules, participates in the transport of mitochondria, APP (amyloid precursor protein) rich vesicle, RNA-containing granules and more. In this case, a set of specific adaptors also dictates the participation of KIF5 in transport to dendrites or to nerve terminals (see section C).

Kinesin based pull-down experiments combined with high coverage mass spectrometry identification is expected to decipher the complexity of the transport molecules and to assess the importance of post-translational 
modifications for the specificity of the kinesin-cargo interactions. A complementary computational effort may rely on sequence-based comparative studies. A sequencebased analysis for identifying simple signatures in the transported proteins for kinesins was not successful [28]. The plant $A$. thaliana exhibits unexpected complexity in term of motor proteins (of course, plants lack neurons). The A. thaliana genome contains as many as 61 kinesins, many of which are still orphan, with no information regarding their movement directionality or their cargo specificity. On the other hand, the sequences of fly, bee, worm, zebrafish, chicken, rodents and other mammalian genomes are already completed. The neuro-anatomical and axonal transport properties in all these organisms are very similar. Consequently, a multiple alignment of the kinesin recognition sequences matched with their transported protein homologues can be used as a basis for defining the key elements in the cargo recognition. A more restricted goal is to classify all kinesins by their recognition preferences. In recent years, machine learning tools were successfully applied in very similar tasks for predicting protein-DNA, protein-protein and proteinsmall ligand interaction rules, so this program seems promising for the study of axonal transport. From experimental point of view, testing the ability of engineered chimeric kinesins to deliver specific cargo is critical for discovering specificity rules in axonal delivery.

\section{B. Neurotransmitter Phenotype Specificity}

Neurons in the CNS are characterized by their "phenotype", defined as the property of the synapse for releasing a specific NT. Each phenotype is associated with a set of proteins that are unique for that NT [29-31]. All the NT phenotype proteins must be fully active at the vicinity of the synapse and thus must be efficiently delivered by fast axonal transport (section A) or by a receptor delivery mechanism at the postsynaptic site [32]. Neurons typically release one specific NT but may respond to a variety of other NTs and modulators. The different site of action for each of the NT-specific molecules is shown in the schematic representation of a synapse (Fig. 2). Each NT can only bind to its cognate receptor types that are located at the postsynaptic membrane (Fig. 2A). It is this specific binding that induces activation of the target cell. Furthermore, binding of NT to auto-inhibitory receptors localized at the presynaptic site control the NT release of that synapse (Fig 2B). Once the released NT binds to its appropriate receptors, it is inactivated and removed either by an enzymatic reaction that breaks down the NT (Fig. 2C) or by NT transporters with high selectivity and high capacity that ensures the uptake of the NT back to the presynaptic site. For example, breaking down acetylcholine (ACh) into choline and acetate is carried out by the potent enzyme (acetylcholinesterase, AChE) that resides at the synaptic cleft. A high affinity transporter allows re-uptake of choline (Fig 2D) back to the nerve terminals from which the ACh was released. For ACh, an additional enzyme is needed to chemically recover the NT from its precursors. This is achieved by a single step reaction carried out by the enzyme Choline acetyltransferase (ChAT) (Fig 2E). The full recovery of the release capacity is still dependent on an additional SV transporter whose function is to efficiently pack the NT into SVs (VAChT) (Fig $2 \mathrm{~F})$. The transmission capacity is then restored, and the synapse is ready for an additional cycle.

How does a neuron, which is characterized by a unique NT phenotype, coordinate the expression of the many proteins which are essential for its identity? The question of gene expression coordination is of course central in any biological complex system. A tight control on the amount of NT production, storage, release and uptake is maintained for each synapse. Herein, we discuss one of the strategies for coordinated gene expression. Our discussion is limited to the cholinergic synapse. Specifically, we focus on two key proteins: (i) the enzyme that produces ACh (called ChAT) and (ii) the transporter responsible to store ACh (called VAChT) in SVs (marked as E, F in Fig. 2).

Based on genomic organization studies in the mouse genome, it was shown that the transporter VAChT is located within the first non-coding intron of the gene for ChAT [33]. The latter has several functional splice variants and multiple promoters [34]. Instances of functionally related genes that are located at the same chromosomal site are known primarily in bacterial genomes. Still this phenomenon of VAChT-ChAT arrangement is referred to as the 'cholinergic locus'. The genomic structure in Drosophila and C. elegans shows a highly conserved organization, where the VAChT is expressed with a common noncoding exon and the coding region of it is a single exon that is completely embedded within the ChAT intron [33]. Interestingly, in all these organisms, a sequence of about 2500 nucleotides preceding the VAChT coding region has been shown to recruit a specific silencer and also to contain a stable RNA folded structure [35]. The tools of the genomic browser [36] allow tracking regions of high sequence conservation without a priori knowledge on functional importance. Inspecting the cholinergic locus region shows that the conservation level for a $2500 \mathrm{nt}$ sequence at the vicinity of the ChAT transcripts is very high (higher than for the coding exons of ChAT, marked as orange empty boxes, Fig. 3). It is expected that some general rules concerning the functional relevance of 'conserved' sequences can be extracted.

The coordination in expression of the cholinergic locus genes is based on their physical gene organization. For other NT phenotypes, alternative mechanism for gene expression coordination is applied. For example, in 


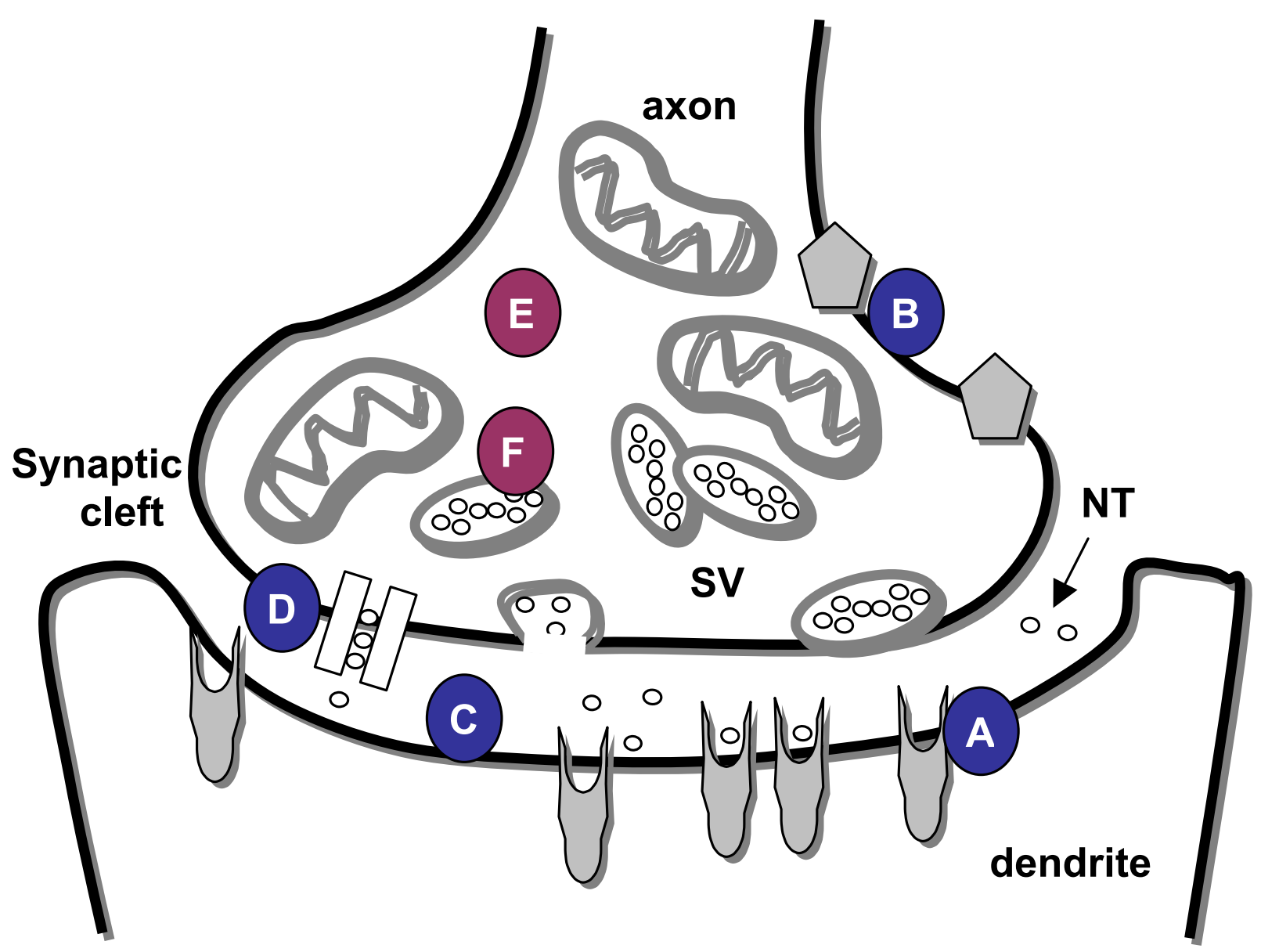

\section{Figure 2}

A schematic view of an ACh synapse. Molecules that specify the NT phenotype are marked A-F. A, a representative of ACh ligand binding receptor family; B, muscarinic ACh receptor as presynaptic autoinhibitors; C, Acetylcolinesterase, AchE; D, Choline high affinity transporter; E, Choline Acetyle Transferase, ChAT; F, Vesicular ACh transporter, VAChT. E and F proteins comprise the 'cholinergic locus' (see text).

GABAergic neurons (the main Inhibitory NT In the brain) a complete coordination between the GABA synthesis enzyme (GAD) and the vesicular GABA transporter is achieved through a shared transcription factor [37].

From a computational perspective, a natural question is to what level the mechanism of 'cholinergic locus' has been duplicated in other context of gene coordination. In more general terms, hidden information in non-coding regions is far richer than previously anticipated. Bearing in mind the phenomenon described above, the presence of small non-coding RNAs and short antisense sequences, a simple assignment of sequences as non-coding should be revised. This fact is in particular critical for the application of machine learning approaches to predict intron-exon boundaries and more recently in the prediction of alternative splicing.

\section{The Dendritic Factory}

Short-term memory in the neuron is executed by modification of already present transcripts and proteins [38]. However, long-lasting forms of memory require macromolecular synthesis [39]. Can long-term memory changes and protein synthesis be demonstrated in only a subset of the synapses in cells that may have thousands of active synapses? To address this question, we should consider the neuronal anatomy. Indeed, expression of specific genes in dendrites is an intriguing idea that can explain specification and variation at the level of individual synapses. The distance of the cell body and the functional 


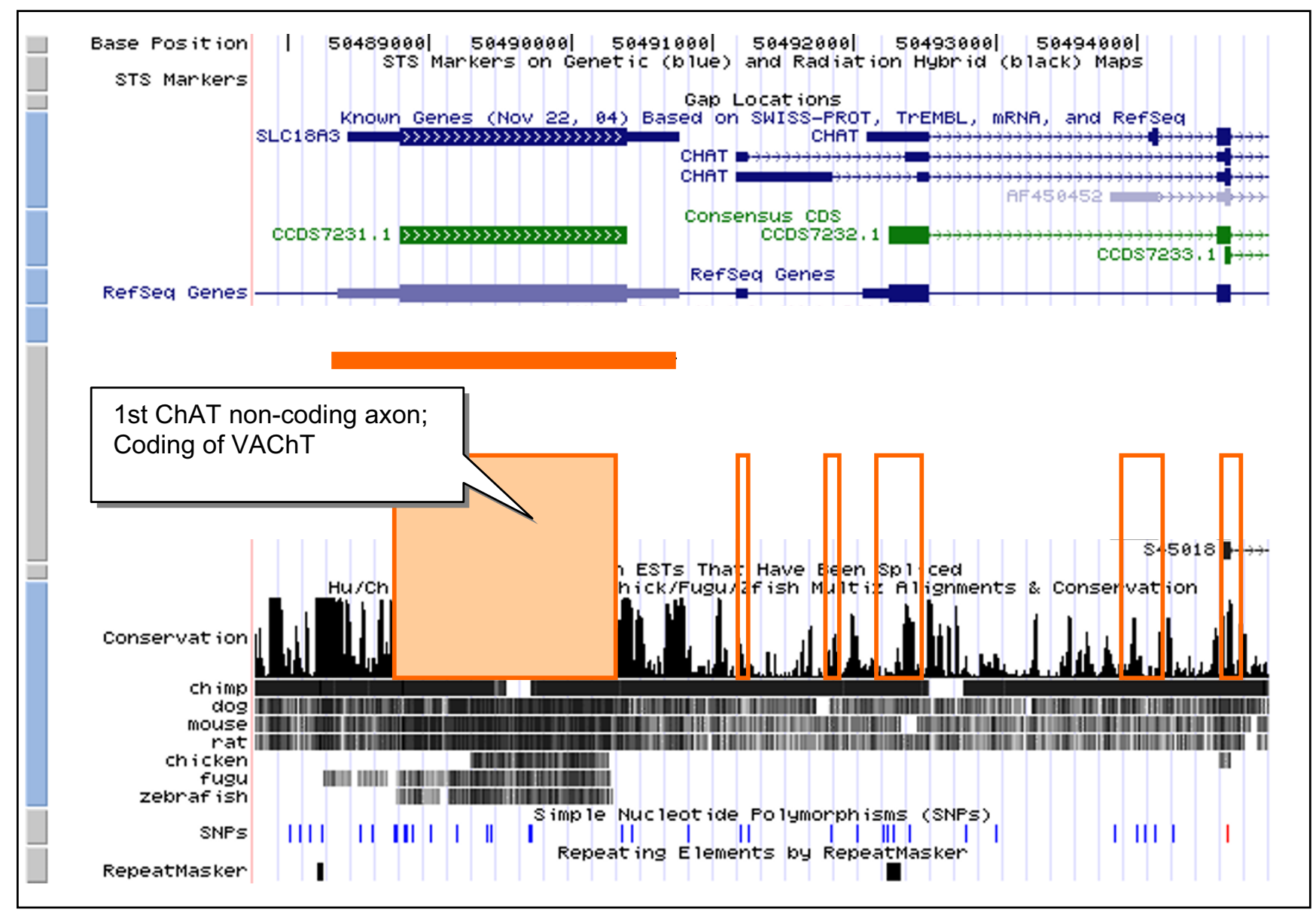

\section{Figure 3}

A visualization and annotation of a genomic region in the vicinity of the 'cholinergic locus' at chromosome 10 (in human). For more information on the visualization, see [36]. Orange lined boxes mark the exons of the ChAT and several of the alternative transcripts. The orange bar indicates the entire length of the first ChAT non-coding exon. The level of conservation among Human-Mouse-Chimp-Rat-Dog-Chicken-Fugu and Zebrafish is shown graphically by the height of the conservation histogram. The pairwise conservation with human is depicted by the scale from black (high) to low (white) for each of the listed organisms. Note that the level of conservation in human-mouse-rat-Fugu extends beyond the coding region of VAChT (semi-transparent orange box).

identity of different domains of the dendritic tree make it unlikely that the protein repertoire is determined by the cell body. In contrast, protein translation at a select dendrite would allow a fast and flexible response to local stimuli [40-42]. This would allow a long-term plasticity at a given synaptic site, in response to changing conditions. For key reviews see [43-47].

The attractive idea of dendritic protein translation is supported by evidence on ribosomes' presence at synaptic junctions of dendrites. A search for RNA at these sites, mostly using in-situ hybridization and amplification techniques, detected several tens of specific mRNA in addition to abundant non-coding RNA of the translation machinery, i.e., rRNA and tRNA $[48,49]$. Later studies established that not only ribosomes but the endoplasmic reticulum and Golgi elements are present in dendrites. In addition, processes of posttranslational modification (i.e phosphorylation) and of membrane insertion were confirmed [50]. A direct illustration of the ability of the dendrite to drive protein translation is based on injecting test mRNAs to dendrites, and monitoring the expressed encoded protein. The subset of RNAs that was shown to accumulate in the synaptic junctions of dendrites includes proteins that were implicated in morphological and functional remodeling of the synapse. Examples are kinases, ion channels and receptors, cytoskeletal elements and proteins involved in trafficking and sorting. Interestingly, some of these selectively delivered RNA that are located in dendrites, become capable of being translated only following 
neuronal activity or neurotrophic action [51,52]. Combining all those observations, one concludes that only stimulated synapses will undergo structural and functional changes that are associated with synaptic plasticity.

Several experiments combining a biochemical purification step followed by PCR-based amplification were performed to resolve the full repertoire of RNAs that can be transported to dendritic sites. The conclusion from these experiments is that the repertoire of RNA that is transported to dendrites is larger than was initially assumed. The most extensive survey using a sensitive PCR based technology argues that 400 different mRNA (about 5\% of the total active transcripts in these neurons) are localized to the dendrites [50]. A more sensitive screening technique suggested a lower bound of $10-15 \%$ of the transcripts as potential candidates for translation regulation [53].

Many unsolved questions remain: (i) What are the shared features (in terms of sequence/structure) of the cis-acting elements that allow RNA molecules to be transported to dendrites? (ii) What is the nature of the repression of RNA that keeps it dormant, allowing it to be activated only following stimulation? (iii) What is the molecular basis for the motor-based delivery of the set of selected RNA?

Some interesting observations from the study of mammalian Staufen (Stau) protein [54] shed light on the trafficking step. Stau is a double-stranded RNA (dsRNA)-binding protein that also contains a microtubule (MT)-binding domain. Thus, Stau may form cross-links between the MT cytoskeletal tracks and RNA components. Despite detailed studies on specific test cases of dendritic RNA, the mechanism for delivering only a subset of RNA to dendrites is not yet fully resolved [55]. It is believed that information encoded in the 3' UTR (untranslated region) of the RNA provide the essential sequence and structure information [56]. Indeed, several of the RNAs known to be delivered to dendrites share a short consensus sequence (called A2RE). Binding of the trans-acting factor (hnRNP-A2) to these sequences will initiate trafficking to dendrites. Furthermore, only RNAs that contain these A2RE-like sequences are assembled into mRNA granules [57]. Those granules contain elements of the translation machinery and the essential MT-based motors. We are still lacking an exhaustive sequence and structure based comparison for hundreds of transported RNAs. Application of feature selection tools and machine learning technologies would help characterize the dendritic transporting specificity.

Once the RNA is delivered to the dendrite, it is kept in a repressed conformation. Only following stimulation, the repression is relieved $[42,43]$. Understanding the sequence of events that lead to activity-dependent relief from the translation arrest is critical to cellular events where regulation at the level of translation dominates. Key players in this switch are a set of activity-dependent kinases that can cause the dormant RNA to undergo a sequential set of changes. The challenge is to build a 'systems-level' model of these events by monitoring the quantities and the states (i.e. phosphorylation) of each of the proteins involved.

Answers to these open questions will be based on developing technologies that allow large scale observations:

\section{(i) Imaging techniques}

By improving the spatial resolution at the neuronal level and monitoring time dependent distribution of essential proteins following activation, one can hope to distinguish between alternative hypotheses for synaptic plasticity.

\section{(ii) Proteomic screening}

A pull-down proteomic survey using one of the components of the mRNA granule allows an unbiased identification of all other components and their composition within the mRNA granules that are delivered to dendrites [58]. Using this approach, it was confirmed that the mRNA granules are composed of tens of proteins including ribosomes, components of the translational machinery as well as several mRNA-binding proteins. Computationally, this requires improving the quality and accuracy of mass spectroscopy based technologies.

\section{(iii) A genomic screen}

An additional proposed regulatory mechanism for controlling localized translation in neurons is by non-coding RNA. An example is the non-coding dendritic RNA BC1 [59] and micro-RNAs (miRNAs) [60]. Tens of complementary sequences in the 3' UTR of target mRNAs were shown to match miRNAs that were associated with polyribosomes in brain extract. A systematic computational survey to characterize the features of the many confirmed miRNAs and to predict others is still missing.

\section{Summary and future perspectives}

We have presented several issues that are critical for the functionality of the synapse - axonal transport, molecular based plasticity and coordination in gene expression. Those examples were selected to indicate that additional experimental and computational research is needed to fully appreciate the mechanisms that are used by the neurons to maintain their functional properties. Powered by genomics, proteomics and bioinformatics, combined with classical neuroscience research, will not only be the basis for constructing accurate simulation of a functioning synapse, but also of a functional neuronal circuit in the brain. 


\section{Abbreviations}

ACh, acetylcholine; AChE, acetylcholinesterase; CNS, central nerve system, MT, microtubule; NT, neurotransmitter; $\mathrm{SV}$, synaptic vesicle; UTR, untranslated region;

\section{Acknowledgements}

We would like to thank Asa Ben-Hur for his suggestions and comments. We are grateful to the Sudarsky Center for Computational Biology (SCCB) of the Hebrew University of Jerusalem. A short version of this paper was presented in the NIPS 2004 computational biology workshop.

\section{References}

I. Matthews G: Cycling the synapse: scenic versus direct routes for vesicles. Neuron 2004, 44:223-226.

2. Montgomery JM, Zamorano PL, Garner CC: MAGUKs in synapse assembly and function: an emerging view. Cell Mol Life Sci 2004, 61:911-929.

3. Goda Y, Davis GW: Mechanisms of synapse assembly and disassembly. Neuron 2003, 40:243-264.

4. Abbas L: Synapse formation: let's stick together. Curr Biol 2003 I3:R25-27.

5. Dubnau J, Chiang AS, Tully T: Neural substrates of memory: from synapse to system. J Neurobiol 2003, 54:238-253.

6. Lee SJ, Hori Y, Groves JT, Dustin ML, Chakraborty AK: The synapse assembly model. Trends Immunol 2002, 23:500-502.

7. Garner CC, Nash J, Huganir RL: PDZ domains in synapse assembly and signalling. Trends Cell Biol 2000, I0:274-280.

8. Davis GW: The making of a synapse: target-derived signals and presynaptic differentiation. Neuron 2000, 26:55I-554.

9. Sejnowski TJ, Koch C, Churchland PS: Computational neuroscience. Science 1988, 24I:|299-1306.

10. Reeke GN Jr, Sporns O: Behaviorally based modeling and computational approaches to neuroscience. Annu Rev Neurosci 1993, 16:597-623.

II. Wolpert DM, Ghahramani Z: Computational principles of movement neuroscience. Nat Neurosci 2000, 3:s 1212-1217.

12. Morris CM, Wilson KE: High throughput approaches in neuroscience. Int J Dev Neurosci 2004, 22:5 I5-522.

13. Marcus K, Schmidt O, Schaefer H, Hamacher M, van Hall A, Meyer HE: Proteomics - application to the brain. Int Rev Neurobiol 2004, 6 I:285-3 I I.

14. Grant SG, Blackstock WP: Proteomics in neuroscience: from protein to network. J Neurosci 2001, 21:8315-8318.

15. Choudhary J, Grant SG: Proteomics in postgenomic neuroscience: the end of the beginning. Nat Neurosci 2004, 7:440-445

16. Zeringue HC, Constantine-Paton M: Post-transcriptional gene silencing in neurons. Curr Opin Neurobiol 2004, I 4:654-659.

17. Marcotte ER, Srivastava LK, Quirion R: cDNA microarray and proteomic approaches in the study of brain diseases: focus on schizophrenia and Alzheimer's disease. Pharmacol Ther 2003, 100:63-74.

18. Schneggenburger R, Sakaba T, Neher E: Vesicle pools and shortterm synaptic depression: lessons from a large synapse. Trends Neurosci 2002, 25:206-2 12.

19. Broadie KS, Richmond JE: Establishing and sculpting the synapse in Drosophila and C. elegans. Curr Opin Neurobiol 2002, 1 2:49|-498.

20. Horton AC, Ehlers MD: Neuronal polarity and trafficking. Neuron 2003, 40:277-295.

21. Craig AM, Banker G: Neuronal polarity. Annu Rev Neurosci 1994, 17:267-310.

22. Hirokawa N, Takemura R: Molecular motors and mechanisms of directional transport in neurons. Nat Rev Neurosci 2005, 6:20I-2I4.

23. Hirokawa N, Takemura R: Kinesin superfamily proteins and their various functions and dynamics. Exp Cell Res 2004, 30l:50-59.

24. Cross RA: The kinetic mechanism of kinesin. Trends Biochem Sci 2004, 29:301-309.

25. Endow SA: Kinesin motors as molecular machines. Bioessays 2003, 25:1212-1219.

26. Dagenbach EM, Endow SA: A new kinesin tree. J Cell Sci 2004, I 1 7:3-7.
27. Thomas N, Imafuku Y, Kamiya T, Tawada K: Kinesin: a molecular motor with a spring in its step. Proc Biol Sci 2002, 269:2363-237I.

28. Kirchner J, Woehlke G, Schliwa M: Universal and unique features of kinesin motors: insights from a comparison of fungal and animal conventional kinesins. Biol Chem 1999, 380:915-921.

29. Goridis C, Brunet JF: Transcriptional control of neurotransmitter phenotype. Curr Opin Neurobiol 1999, 9:47-53.

30. Black IB, Patterson PH: Developmental regulation of neurotransmitter phenotype. Curr Top Dev Biol 1980, I5:27-40.

31. Weiler IJ, Irwin SA, Klintsova AY, Spencer CM, Brazelton AD, Miyashiro K, Comery TA, Patel B, Eberwine J, Greenough WT: Fragile X mental retardation protein is translated near synapses in response to neurotransmitter activation. Proc Natl Acad Sci U S A 1997, 94:5395-5400.

32. Sheng M: Molecular organization of the postsynaptic specialization. Proc Natl Acad Sci U S A 200I, 98:7058-706I.

33. Erickson JD, Weihe E, Schafer MK, Neale E, Williamson L, Bonner TI, Tao-Cheng JH, Eiden LE: The VAChT/ChAT "cholinergic gene locus": new aspects of genetic and vesicular regulation of cholinergic function. Prog Brain Res 1996, 109:69-82.

34. Oda Y: Choline acetyltransferase: the structure, distribution and pathologic changes in the central nervous system. Pathol Int 1999, 49:921-937.

35. De Gois S, Houhou L, Oda Y, Corbex M, Pajak F, Thevenot E, Vodjdani G, Mallet J, Berrard S: Is RE I/NRSE a common cis-regulatory sequence for ChAT and VAChT genes? J Biol Chem 2000, 275:36683-36690.

36. Karolchik D, Baertsch R, Diekhans M, Furey TS, Hinrichs A, Lu YT, Roskin KM, Schwartz M, Sugnet CW, Thomas DJ, et al.: The UCSC Genome Browser Database. Nucleic Acids Res 2003, 3 I:5 I-54.

37. Schuske $K$, Beg AA, Jorgensen EM: The GABA nervous system in C. elegans. Trends Neurosci 2004, 27:407-4I4

38. Bliss TV, Collingridge GL: A synaptic model of memory: longterm potentiation in the hippocampus. Nature 1993, 36I:3I-39.

39. Goelet P, Castellucci VF, Schacher S, Kandel ER: The long and the short of long-term memory-a molecular framework. Nature 1986, 322:419-422

40. Smith WB, Aakalu G, Schuman EM: Local protein synthesis in neurons. Curr Biol 200I, I I:R90I-903.

41. Eberwine J: Molecular biology of axons: "a turning point...". Neuron 2001, 32(6):959-960.

42. Steward O, Schuman EM: Protein synthesis at synaptic sites on dendrites. Annu Rev Neurosci 200I, 24:299-325.

43. Kelleher RJ 3rd, Govindarajan A, Tonegawa S: Translational regulatory mechanisms in persistent forms of synaptic plasticity. Neuron 2004, 44:59-73.

44. Wang $\mathrm{H}$, Tiedge $\mathrm{H}$ : Translational control at the synapse. Neuroscientist 2004, I 0:456-466.

45. Mohr E: Subcellular RNA compartmentalization. Prog Neurobiol 1999, 57:507-525.

46. Job C, Eberwine J: Localization and translation of mRNA in dendrites and axons. Nat Rev Neurosci 200I, 2:889-898.

47. Richter JD: Think globally, translate locally: what mitotic spindles and neuronal synapses have in common. Proc Natl Acad Sci U S A 200I, 98:7069-707I.

48. Miyashiro K, Dichter $M$, Eberwine J: On the nature and differential distribution of mRNAs in hippocampal neurites: implications for neuronal functioning. Proc Natl Acad Sci U S A 1994, 91:10800-10804

49. Crino PB, Eberwine J: Molecular characterization of the dendritic growth cone: regulated mRNA transport and local protein synthesis. Neuron 1996, I7: I I73-I I87.

50. Eberwine J, Miyashiro K, Kacharmina JE, Job C: Local translation of classes of mRNAs that are targeted to neuronal dendrites. Proc Natl Acad Sci U S A 2001, 98:7080-7085.

5I. Klann E, Antion MD, Banko JL, Hou L: Synaptic plasticity and translation initiation. Learn Mem 2004, I I:365-372.

52. Zhang $X$, Poo MM: Localized synaptic potentiation by BDNF requires local protein synthesis in the developing axon. Neuron 2002, 36:675-688

53. Pradet-Balade B, Boulme F, Beug H, Mullner EW, Garcia-Sanz JA: Translation control: bridging the gap between genomics and proteomics? Trends Biochem Sci 2001, 26:225-229. 
54. Roegiers F, Jan YN: Staufen: a common component of mRNA transport in oocytes and neurons? Trends Cell Biol 2000, 10:220-224.

55. Richter JD: RNA transport (partly) revealed! Neuron 2004, 43:442-443.

56. Rook MS, Lu M, Kosik KS: CaMKIlalpha 3' untranslated regiondirected mRNA translocation in living neurons: visualization by GFP linkage. J Neurosci 2000, 20:6385-6393.

57. Shan J, Munro TP, Barbarese E, Carson JH, Smith R: A molecular mechanism for mRNA trafficking in neuronal dendrites. J Neurosci 2003, 23:8859-8866.

58. Angenstein F, Evans AM, Ling SC, Settlage RE, Ficarro S, Carrero-Martinez FA, Shabanowitz J, Hunt DF, Greenough WT: Proteomic characterization of messenger ribonucleoprotein complexes bound to nontranslated or translated poly(A) mRNAs in the rat cerebral cortex. J Biol Chem 2005, 280:6496-6503.

59. Zalfa F, Giorgi M, Primerano B, Moro A, Di Penta A, Reis S, Oostra $B$, Bagni $C$ : The fragile $X$ syndrome protein FMRP associates with BCI RNA and regulates the translation of specific mRNAs at synapses. Cell 2003, I I 2:3 |7-327.

60. Kim J, Krichevsky A, Grad Y, Hayes GD, Kosik KS, Church GM, Ruvkun G: Identification of many microRNAs that copurify with polyribosomes in mammalian neurons. Proc Natl Acad Sci USA 2004, 101:360-365.

Publish with Bio Med Central and every scientist can read your work free of charge

"BioMed Central will be the most significant development for disseminating the results of biomedical research in our lifetime. "

Sir Paul Nurse, Cancer Research UK

Your research papers will be:

- available free of charge to the entire biomedical community

- peer reviewed and published immediately upon acceptance

- cited in PubMed and archived on PubMed Central

- yours - you keep the copyright

Submit your manuscript here:

http://www.biomedcentral.com/info/publishing_adv.asp 